\title{
Predicting Safety Related Attitudes in the Workplace: The Influence of Moral Maturity and Emotional Intelligence
}

\author{
Francis L. Jeffries \\ University of Alaska, Anchorage
}

\begin{abstract}
Safety in the workplace has received an increasing amount of attention for over 50 years. Approaches to improving safety have focused primarily on extrinsic drivers such as engineering safer work environments, training, and policies. These efforts have reduced injuries and reportable incidents. Yet, progress toward industry's stated goal of zero incidents has stalled. This paper explores an approach to creating a safer work environment using intrinsic drivers instead of compliance oriented behaviors. The Theory of Planned Behavior (Ajzen, 1985) is employed to show that moral maturity and emotional intelligence can act as intrinsic drivers, positively influencing safety attitudes and behavior.
\end{abstract}

\section{Introduction}

Working in a safe environment is of great importance to employees, industry, and government. Two government agencies and an entire industry in the private sector are devoted to helping improve safety in the workplace. Reducing the human and financial costs associated with unsafe operation and eliminating accidents is now part of most, if not all, organizations' strategic goal set.

Early efforts at increasing safety addressed reducing risk at the human interface through engineering and training (Ellis, 1975). Later approaches used observation and feedback (Weinstein, 1998) and more recently others have called for "actively caring", that is, being concerned about another and doing something about it (Geller \& Wiegand, 2005). All of the approaches have made progress by reducing accidents, though none have yet been successful reaching the stated goal of zero accidents.

Studies to this point have focused primarily on extrinsic processes involved in creating a safe environment. The result is that there has not been adequate attention paid to the moral imperative to behave in a safe manner or the intrinsic side of safe behavior. Two potentially promising influences on safety related attitudes leading to safer behaviors are an individual's moral maturity and Emotional Intelligence (El). Moral maturity is an individual's cognitive ability to reason through the information surrounding a situation and make an ethical decision. El is an individual's ability to understand the context of a particular decision and to decide what to do based on an understanding and appreciation of the potential consequences of the decision for oneself and others. It has been shown that training increasing moral maturity has positive effects on the behavior of people in business (Jones \& Ryan, 1997; Wulfson, 1998). This is 
presumably because of the positive impact on attitudes. El has also been shown to have an effect on performance in organizational contexts (Van Rooy \& Viswesvaran, 2004) and this is presumably because it has a positive impact on attitudes related to performance (though there is no known research expressly investigating the El-attitude link).

Moral maturity is a central construct found in ethics research as it relates to predicting behavior. A search was conducted to find research identifying linkages between ethical behavior and safety-related behavior. While there was no research specifically linking moral maturity and safety, research connecting ethical climate and its effect on safety behavior was found. Parboteeah and Kapp (2008) discussed the effect of ethical climate on safety performance and concluded that an organization's ethical climate is associated with compliance with safety-related behaviors. In their initial research on the relationship of ethical climate to safety-related behaviors, Parboteeah and Kapp (2008) found that organizations with a principled ethical climate demonstrated higher levels of compliance with both wearing protective equipment and membership on safety committees. While this demonstrates a potential link between ethics and safety-related behavior, the research was focused on the ethical climate of the organization as opposed to the ethics of the individuals involved. Other researchers have theorized about organizational ethical climate and its impact on safety-related behaviors (Tidwell, 2000 ) and the organization's obligation to provide a safe workplace as a moral responsibility (Angelini, 1987; Birkner \& Birkner, 2000; Eckhardt, 2001). McKendall, DeMar, and Jones-Rikkers (2002) argued that ethics programs (rules, training, etc.) should lead to fewer OSHA violations. So there is evidence that ethical behavior and safety are related at the organizational level of analysis. This logically leads to the conclusion that they are likely to be related at the individual level of analysis too. That being the case, the role of ethical behavior on safety-related attitudes and behavior should be investigated.

At the individual level of analysis ethical behavior has been shown to be influenced by moral maturity as defined by the P score (principled score) on the Defining Issues Test (DIT). Rest (1994) reported that there were over 1,000 studies using the DIT at that time and there is also considerable support establishing the connection between moral maturity and behaving ethically (e.g. Abdolmohammadi \& Sultan, 2002). Since it has been established that ethical climate influences safety-related behavior (Martin \& Cullen, 2006) it is reasonable to expect that moral maturity, leading to more ethical behavior, has potential to be a positive influence on safety-related attitudes as well.

There are two conceptualizations of El currently used in the literature. One is an ability model that sees El as a kind of intelligence (Mayer, Salovey, \& Caruso, 1999). The other is called the mixed model, a trait-based approach (Goleman, 1999). El involves empathy (Goleman, 1999) and empathy has been closely linked to social support and altruistic behavior (Huy, 1999). Other dimensions of El have been described as selfregulation (Goleman, 1999), consequential thinking, and pursuit of noble goals (Freedman, 2002). In the context of safety, all of these should yield a positive influence on attitudes toward safety. That is, thinking before acting, considering possible actions 
and making decisions that will result in positive outcomes, and making decisions that are in concert with one's goals and principles should all be supportive of positive attitudes toward safe behavior of oneself and others.

What follows is an explanation of why moral maturity and El will have a positive influence on safety-related attitudes. First a brief history of the safety culture movement will be offered followed by a section on moral maturity and then one on El. Next, the connections between moral maturity and safety and El and safety will be developed along with propositions. Finally the paper will conclude with a summary and suggested future directions.

\section{Safety Culture}

There is a substantial amount of interest in the creation of a safer workplace. Entire journals, for example Professional Safety, are dedicated to the topic. Others regularly publish research on the topic of safety such as Journal of Construction Research, Engineering Management Journal, and Journal of Healthcare Management. There are a variety of reasons for the interest in creating safer work environments, one of them being the high cost of accidents. The Occupational Health and Safety administration (OSHA) estimates that each fatality results in costs up to $\$ 910,000$ and each prevented illness or injury avoiding lost work days would save $\$ 28,000$ (Cowie, 2002).

The earliest attempts at improving safety focused on engineering a safer work environment, educating employees regarding use of engineering interventions, and enforcing the new work practices with discipline (Geller, 2001). Removing hazards and re-engineering work areas to eliminate obvious high risk human interfaces resulted in nearly immediate positive outcomes for both the workers and the organization. While this approach was effective to a point, it only addressed the physical work environment. The lack of a behavioral focus seems odd considering that Heinrich (1959) estimated that $88 \%$ of all industrial accidents were caused by unsafe acts. Over time it became clear that enforcing discipline and requiring compliance can achieve only limited success when creating a safer work environment. As noted by Weyman, Clarke, and Cox (2003), engineering solutions to safety issues can only go so far and the rest is up to "...human behavior, specifically error and risk-taking" (p. 307).

Among those researchers who took the lead in calling for and researching a behavioral approach to improving safety practices in industry were Fitch, Hermann, and Hopkins (1976), Reber, Wallin, and Chhokar (1984), and Smith, Anger, and Ulsan (1978). In an intervention typical of the early attempts at changing behavior Reber et al. (1984) used observations, goal setting, and feedback in a controlled experiment at an industrial plant. In this case they achieved an increase in targeted safe behaviors from a baseline of $61.5 \%$ to $95.4 \%$ in 1981 . This translated to a reduction in lost-time injuries of nearly $54 \%$ and the overall injury rate dropped by nearly $35 \%$.

The current industry approach to safety that is largely accepted is Behavior Based Safety (BBS). It had its beginnings in three separate, but similar, efforts by Komaki, Barwick, and Scott (1978) (academicians) who were researching applied behavioral 
analysis, Dr. John Hudley who was working for a petrochemical company, and Gene Earnest and Jim Palmer then working for Proctor and Gamble who reportedly coined the phrase "Behavior Based Safety" (Krause, 2001). In a study analyzing the effectiveness of BBS, 31 of 32 research articles reported a reduction in injuries (SulzerAzaroff \& Austin, 2000).

Throughout this evolution of the practice of creating a safer work environment the focus has been on three primary areas; the work environment, the people involved, and behavior (Geller \& Wiegand, 2005). BBS efforts have attempted to change employee at risk behaviors to safe behaviors primarily through employee training, observation, and feedback. As noted by Geller and Wiegand (2005) these attempts have been quite effective at gathering the "low hanging fruit" resulting in commendable improvements in safety. However, just as attending to environmental issues alone to improve safety achieved good results and then plateaued, BBS efforts are running into the same phenomenon. The leading edge companies are now looking for the next answer to the question "how do we improve from here?" (Geller \& Wiegand, 2005).

One of the current directions being taken in the field is to examine personality traits that will predict who will and not be safe (Geller \& Wiegand, 2005). Presumably this will allow organizations to identify those who may be more likely to be predisposed to have favorable attitudes toward safety and safe behaviors. This predisposition, they argue, will have a positive impact on the person's desire to perform work in a safe manner resulting is safer workplace behaviors and thus, fewer incidents.

To date, the author found only one study that has examined the relationship between the big five personality traits (openness to new experience, conscientiousness, extroversion, agreeableness, and neuroticism) and safety. Cellar, Nelson, \& Bauer (2001) used self-report measures of the NEO-I-PR and self-report measures of prior workplace incident involvement. They found that there was a significant inverse relationship between agreeableness and the total number of reported workplace accidents and between conscientiousness and the total reported not-at-fault work related accidents as well as the total reported work-related accidents.

While the progress toward creating a safe work environment to date is laudable, it seems that none of the interventions have specifically focused on lasting changes to critical intrinsic factors determining human behavior, namely affective and moral influences on attitudes toward safety. The theory of planned behavior (Ajzen, 1985) describes three influences antecedent to a person's behavior. These are attitude toward the act, subjective norms, and perceived behavioral control. Previous efforts at creating a safe work environment have focused on creating subjective norms compatible with safe work practices through the observation and feedback process. Perceived behavioral control has been influenced by the assurance that unsafe behaviors will be noted and discipline will be applied to correct them as needed. It is possible that attitudes toward safe behavior may have been impacted by training and requirements for compliance to rules and regulations. However, it is just as likely that much of safetyrelated behavior is compliance-based rather than truly voluntary behavior. Subjective 
norms and the need to conform to rules and policy are extrinsic reasons for compliance to safety requirements. If there were intrinsic drivers, true motivation to be safe, it is likely that it would result in fewer reportable incidents.

This paper asserts that improving workers' moral maturity and/or El is an alternate way to positively impact safety attitudes and behavior. The rationale, more fully developed below, is that people with a more altruistic, as opposed to egocentric, set of values will be more likely to demonstrate safe work behaviors. Those at a principled level of moral maturity and those at higher levels of El will consider consequences for themselves and others before acting. This is consistent with a favorable attitude toward safety and safe behavior and it represents an intrinsic driver providing motivation to be safe.

\section{Moral Maturity}

Kohlberg (1969) developed a six stage model of cognitive moral development that is well accepted and still widely used today. His model has 6 stages as described below. The stage of punishment and obedience (stage 1) is an approach to moral behavior that is literal obedience to the letter of the rules and authority. There is no association of morality with any higher order or philosophy, but rather to the person who has power. The stage of individual instrumental purpose and exchange (stage 2) is where that which serves one's own needs defines moral behavior. Rules and authority take a back seat to what is fair to the individual (in his/her own estimation) at this stage. Decisions are made on the basis of a negotiated outcome that benefits the individual and may or may not conform to the guidelines of the authorities. The stage of mutual interpersonal expectations, relationships, and conforming (stage 3 ) is where the individual begins to emphasize others rather than him/herself. Obedience to the rules is still a primary motivator here, but the well-being of others now plays a larger role in the morality of decisions being made. The primary difference between this stage and the preceding one is the consideration of others in making the decision. The stage of social system and conscience maintenance (stage 4) is where the individual considers his/her responsibility to society not just other specific people. One has a sense of duty, respect for authority, and maintaining social order driving moral behavior at this stage. The stage of prior rights and social contract or utility (stage 5) is where decisions are made based on concern for upholding some basic rights, values, and legal contract with society. Moral behavior here involves a sense of obligation or commitment and an awareness of a "social contract" to/with other groups. The final stage is the stage of universal ethical principles (stage 6) where one believes that there are universal ethical principles and that everyone should follow them. Laws, rights, and social agreements are not valid because of a society's law or custom, but rather because they rest on universal principles. At this stage, one's ethical practice does not rely on an organization or society for ethical direction; s/he relies on universal principles such as justice and equality to determine the morality of a given behavior.

To simplify the discussion of the model it has also been presented as 3 stages: preconventional (stages 1 and 2), conventional (stages 3 and 4), and postconventional or principled (stages 5 and 6). Those in the preconventional stage are focused mainly 
on what is good for oneself and conformity to rules to avoid punishment. Those in the conventional stage show an awareness of other's opinion of their behavior and act in ways to fit in. Those in the postconventional stage act in a principled manner in conformity to universal principles of moral behavior regardless of laws, rules, or consequences.

Moral maturity, as measured by the Defining Issues Test (DIT), yields a P score of the respondent's moral reasoning ability (Rest, 1993). The P score is short for the Principled score. It is an indication of moral development with higher scores representing higher levels of moral development. In Rest's words, the P score represents: "the degree to which a person's thinking is like that of moral philosophers" (Rest, 1993, p. 13). The DIT is based on Kohlberg's (1969) model described above. The DIT has been used extensively in the area of business ethics with no fewer than 50 studies published in the Journal of Business Ethics from 1996 to 2005 (Herrington \& Weaven, 2008).

Higher P scores on the DIT relate to greater cognitive reasoning ability regarding ethically challenging situations. Actions of those with higher $P$ scores have been associated with more ethical behavior in numerous situations. For example, in stock trading simulations with insider information, those with higher $\mathrm{P}$ scores were less likely to use the information to their advantage (Abdolmohammadi \& Sultan, 2002). Wright (1995) also discussed the effects of environmental factors on ethical behavior and notes that persons with an external locus of control are more susceptible to environmental factors [subjective norms and perceived behavioral control for example, using Ajzen's (1985) model] and are more likely to behave in a manner inconsistent with their moral judgment. Jones and Ryan (1997) argued that there are several influences on a person's decision to act ethically (or not). The factors they noted are: severity of the consequences of the act, certainty that the act is moral or immoral, the actor's degree of complicity in the act, and the pressure the person feels to act unethically. The actor weighs these factors and if the level of approbation (approval or praise in this case) meets the threshold, then the person performs the act. Peer and/or authority approval is the lynch pin in this situation as opposed to the morality of the act. This also is consistent with the theory of planned behavior (Ajzen, 1985) and its components, subjective norms and perceived behavioral control.

In other research on employees in the public sector, White (2002) found that situational characteristics are influential. In particular he identified presence of an authority responsible for consequences, remoteness of victims, subordinate roles, and binding rules of protocol and etiquette. He concluded that public managers generally make decisions based on rules as opposed to individual discretion and that dynamic consistency (adherence to moral standards in decision-making) can be overridden by external influences. This is consistent with Jones and Ryan (1997) and their observations regarding managers in the private sector.

The majority of managers are at level 3 or 4 (level 3: mutual interpersonal expectations, relationships, and conforming and level 4: social system and conscience maintenance) 
of Kohlberg's (1969) moral maturity hierarchy. If more were at level 5 and 6 (level 5: prior rights and social contract and level 6: universal ethical principles) compliance to the rules would presumably not be the driver, doing the right thing because it is the right thing to do would be the driver. That is, one would be acting based on character of integrity. So, while there is evidence that moral maturity is generally a positive influence on ethical behavior there is also evidence that situational factors are influential as well.

The prevailing model for predicting ethical behavior to this point has been one based on cognitive moral development as measured by the DIT. Cognitive ability has been shown to be related to job performance, yet it only explains about $25 \%$ of the variance (Goldstein, Zedeck, \& Goldstein, 2002; Hunter \& Hunter, 1984). As noted by Van Rooy and Viswesvaran (2004) in a recent meta-analysis of El research, the most successful employee will likely have high level of both general mental ability and emotional intelligence. Applying this line of reasoning to decision making relative to safety, the inclusion of a construct that represents the affective side of decision making has the potential to increase our understanding of safety-related behavior and may increase our ability to more accurately predict who will practice safe behavior. Emotional Intelligence is a construct that can account for the affective side of the ethical decision-making process.

\section{Emotional Intelligence}

There has been a substantial amount of research reported regarding El in the management literature over the past 20 years. A search of EBSCO's Business Source Premier, on January 10, 2011, using the key words "emotional intelligence" resulted in a list of 545 journal articles. There have been specialized tracks in conferences, special issues of journals, and books focused on El. Claims have been made in both the practitioner literature and empirical studies that El has a positive influence on effective leadership, learning in classes, and performance on the job. This has resulted in interest among human resource professionals who are using $\mathrm{El}$ as a tool for personnel hiring and in training (Fineman, 2004).

There are currently two schools of thought regarding El as a construct. One is the ability model defining El as a type of intelligence (Mayer, Salovey, \& Caruso, 1999) measured by the Multifactor Emotional Intelligence Scale (Mayer, Caruso, \& Salovey, 1999). The other is the mixed model in which El is seen as more dispositional or trait-based (Petrides \& Furnham, 2001) and it its typically measured with the Emotional Quotient Inventory (Bar-On, 2000). There is quite a debate going on regarding the efficacy of the respective models. While some are adamant in support of their favorite conceptualization of El and its measurement (e.g. Brackett \& Mayer, 2003; Bar-On, 2000) others see them as complimentary (Ciarrochi, Chan, \& Caputi, 2000).

Regardless, there has been a substantial amount of empirical research done using both conceptualizations and both models have acceptable psychometric properties and predictive ability and therefore are useful in organizational settings (Van Rooy, Viswesvaran, \& Pluta, 2005). 
El is 'the ability to monitor one's own and others' feelings and emotions, to discriminate among them and to use this information to guide one's thinking and actions" (Salovey \& Mayer, 1990: 189). El is distinct from other forms of intelligence, general intelligence for instance, since it is involved specifically with the management of emotions and emotional content (Jordan, Ashkanasy, Härtel, \& Hooper, 2002; Mayer \& Salovey, 1997). The focus on recognizing and managing emotion makes El distinct from impression management (Lennox \& Wolfe, 1984; Snyder, 1979).

Mayer \& Salovey (1997) developed a four component model of El as an ability. Emotional perception is the ability to be self-aware of emotions and to be able to express emotions and emotional needs accurately to others. Emotional assimilation is the ability of one to distinguish among the different emotions he/she may be feeling and to prioritize those that are influencing his/her thought processes. This allows one to focus on important information and to understand why the feelings are being experienced. Emotional understanding is the ability to understand complex emotions such as simultaneous feelings of loyalty and betrayal. Emotion management is the regulation of one's emotions. This involves the ability to connect with or to disconnect from a given emotion depending on its appropriateness for a given situation.

Goleman (1999), who takes the trait approach, asserts that one with emotional intelligence is self-aware, able to recognize and understand one's moods, emotions, drives, and their effect on others. They are able to control or redirect disruptive reactions before they act out and have the ability to think before acting while suspending judgment. They display the ability to show empathy, that is, to understand the emotional makeup of others, and use this skill in behaving toward others appropriately according to their emotional reactions. Empathy underlies the success of social support and is closely associated with altruistic behavior (Huy, 1999). Those high in El also possess social skills that promote proficiency in relationship and network building, the ability to find common ground, and to build rapport.

In sum, El is the ability to be aware of one's own emotional state, to regulate those emotions, and to respond to the situation that is the catalyst of the emotion in a way that facilitates acquisition of outcomes that are sought by the individual. The value of $\mathrm{El}$ in organizational life is actively being researched and has shown to be related positively to both personal and organizational outcomes as described below.

Positive outcomes related to El were found by Jordan, et al. (2002) who concluded that employees who scored higher on El assessments would be less likely to adopt negative coping strategies in response to stress related to job insecurity. Negative coping strategies are reactive and defensive and are associated with a reduction in organizational commitment. Lam \& Kirby (2002) found that El explained performance beyond that which was attributable to general intelligence. Specifically, they found that overall El, perceiving emotions, and regulating emotions all made contributions to performance that were distinct from general intelligence's contribution. Abraham (2000) observed that individuals with high El were able to set priorities and attend to tasks that are of a higher priority. In the work setting, she related this to the need for control over 
the job so that employees could allocate their time most efficiently setting new goals. Furthermore, allowing the employee this freedom had a positive impact on job satisfaction and commitment.

In work settings, El facilitates addressing the issues rather than taking the politically correct approach. This could result in job loss in a repressive environment but where there is sufficient job control it creates space for freedom of choice and expression (Abraham, 2000). Benefits of El are higher commitment, greater job satisfaction, and resilience in the face of difficult situations. Additionally, performance improvements attributable to El have been reported by numerous researchers (Cooper \& Sawaf, 1997; Goleman, 1995; Lam \& Kirby, 2002; Salovey \& Sluter, 1997; Weisinger, 1997). Finally, in a meta-analytic report covering 69 studies regarding El and performance Van Rooy and Viswesvaran (2004) found a .23 correlation between El and performance. If similar results can be duplicated linking $\mathrm{El}$ and safety attitudes it will have significant implications for the field. Next, the rationale linking safety attitudes to moral maturity and El will be presented.

\section{Moral Maturity, El, and the Connection to Safety}

In a previous discussion, safe behavior was said to be supported by engineering a safer work environment and educating employees (Geller, 2001) and creating opportunities for employees to be observed and receive feedback useful for improving safety (Fitch, et al. 1976; Geller, 2001; Geller \& Wiegand, 2005; Reber, et al. 1984; Smith, et al., 1978). Now there is a move to develop tools to predict which employees (or job candidates) will be predisposed to behave in a safe manner (Cellar, et al., 2001; Geller \& Wiegand, 2005). All of these efforts have had a positive impact on creating a safer work environment and reducing accidents, lowering the number of days lost to injuries, and reducing fatalities. However, the stated goal of the safety programs is to get to zero, that is, no reportable accidents/incidents. In order to do this it will be necessary to go beyond previous and current initiatives to improve safety and address the individual worker's attitudes about working safe, their own safety, and other's safety.

Putting it in terms of Ajzen's (1985) theory of planned behavior, the previous attempts have correctly targeted perceived behavioral control through training, engineering, and compliance measures using discipline and sanctions to ensure results and to make sure that procedures are followed. Subjective norms have been influenced through efforts to create a safety culture and through the process of observation and feedback. This creates a common cultural approach toward safety and creates peer pressure to conform to safety policies. While it can be argued that these previous efforts have influenced attitudes (positive behavioral results have been observed and this is prima facie evidence that attitudes toward the safety act in question were positive) no real effort has been focused at understanding enduring intrinsic influences on safety attitudes. Compliance to policy, due to the possibility of sanctions for non-compliance or peer pressure to conform, is an extrinsic influence on behavior. In order to achieve further improvements in enduring safety attitudes at work an intrinsic drive to create a 
safe workplace will be helpful. Moral maturity and El have potential to be enduring positive intrinsic influences on safety attitudes.

Moral maturity will have effects on decision making regarding safety-related behaviors from a cognitive perspective (judgment driven behaviors) as opposed to $\mathrm{El}$ and its affective influence. As discussed earlier, efforts have been made to create rules and consequences for safe behavior (Geller, 2001). There have also been efforts directed at creating a culture of safety and to encourage safe behavior through observation and feedback (Geller \& Wiegand, 2005). These programs, when examined in the context of the theory of planned behavior address perceived behavioral control and subjective norms. Having policies that prescribe consequences for failure to follow safe work practices and providing training and equipment influences perceived behavioral control. There is less freedom to engage in unsafe practices because of the likelihood of adverse personal outcomes and because individuals have the skills and tools to meet expectations related to safe behavior. Creating a culture of safety and having peers observe each other and provide feedback create subjective norms favoring safety that will increase the likelihood the individual will intend to behave in a safe manner.

The three levels of moral maturity; preconventional, conventional, and principled can be seen as generally relating to the actions taken by industry to improve safety. The early engineering efforts and training relate to the preconventional level of moral maturity in that compliance is all that really matters and it does not really require higher order thinking or moral maturity to capture improvement in safety. Behavior Based safety with its attendant awareness of others, observations and feedback, etc. (Geller \& Wiegand, 2005 ) is related to the conventional level of moral maturity since it involves social awareness and responsibility to others. The principled level has not yet been addressed directly and this is where the potential for improvement is now the greatest. If employees can be convinced to do the right thing as it relates to safe behavior, regardless of the rules (or lack of them) or the situation it will be a real breakthrough. 
Figure 1. Theory of Planned Behavior with El and Moral Maturity added

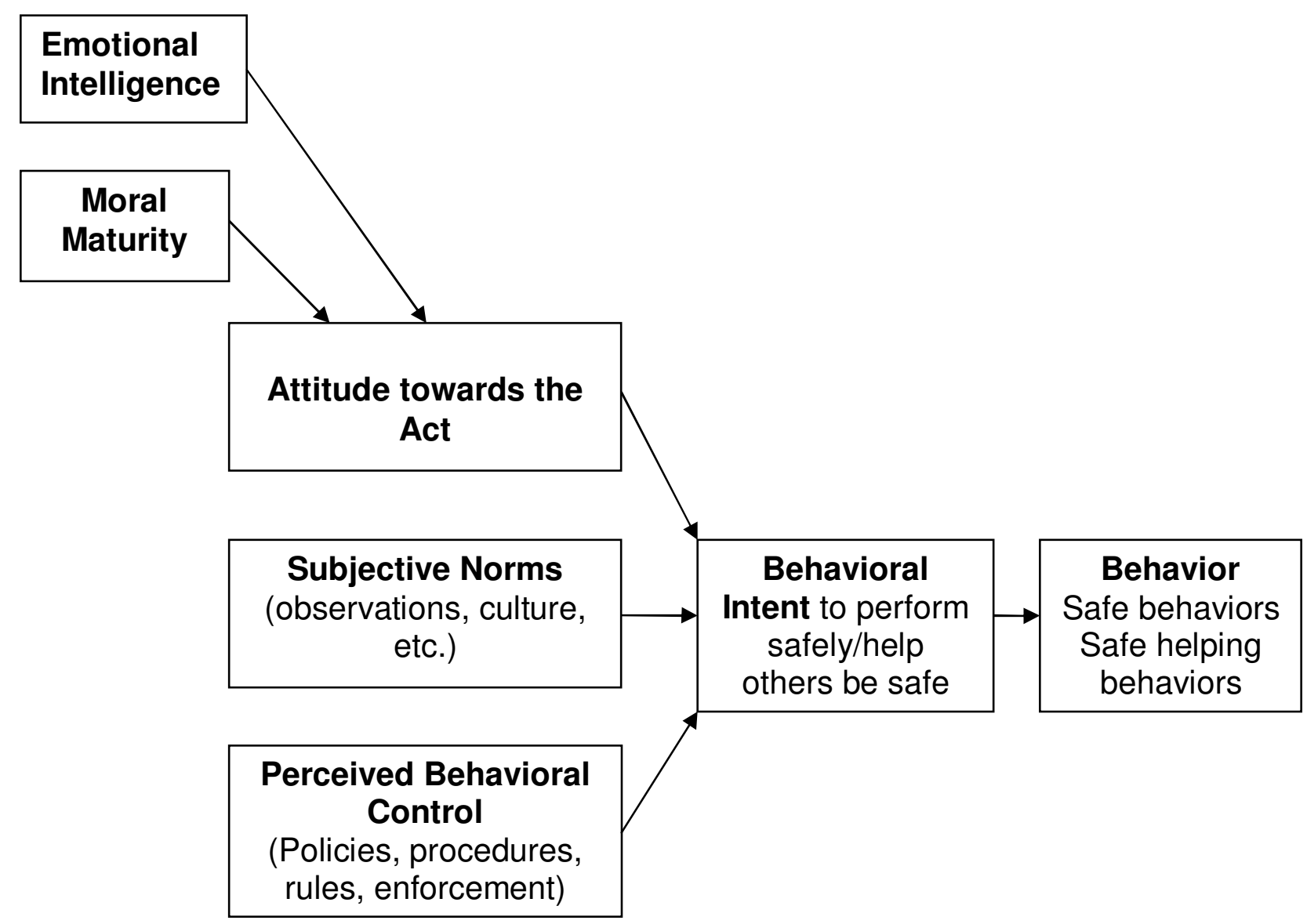

an individual is at the preconventional level of moral maturity, it is unlikely that an employer will attain more than compliance with the rules relating to safety. This will be ethical and moral in the employee's eyes at this stage and it will not be necessary or desirable to do more. If an individual is at the conventional stage, being involved in the tool box meetings and being on safety committees will be seen as reasonable and expected as long as the employee's peer group is engaged in the same activities. Having a safety culture would facilitate creation of norms favorable to being engaged at this level. If an individual is at the principled level, s/he is guided by values and universal principles. This person will perform safe behaviors regardless of the circumstances because it is the right thing to do. Thus:

Proposition 1: Persons with greater moral maturity will have more favorable attitudes toward safe working practices than those with lower moral maturity. 
Proposition 2: Persons with greater moral maturity will consistently perform more safely than those with lower moral maturity.

The qualities of a person high in El are not attitudinal in nature. They are more like an affective skill set: emotional perception, emotional assimilation, emotional understanding, and emotion management (Mayer \& Salovey, 1997) or traits: empathy, optimism, motivation, ability to engage in relationships (Goleman, 1995). These in turn affect work attitudes and attitudes impact behavior through behavioral intent (Ajzen, 1985 ) resulting in positive or negative judgment-driven behaviors (quitting, anti-, or prosocial behaviors) and/or positive or negative affect-driven behaviors (impulsive acts or helping) (Weiss \& Cropanzano 1996). Emotional intelligence has an effect on the process through its action as an influence on the experienced emotions potentially limiting negative affect-driven behaviors and dampening adverse situational impacts on attitudes.

In other work relevant to the impact of El on behavior Lam and Kirby (2002), while discussing the process El involves, describe the emotional control mechanisms buffering and personal engagement. Buffering involves isolating emotions so that they do not interfere with the task one is performing. Personal engagement, the emotional involvement in tasks, represents the highest level of motivation resulting in high performance. This has been compared to flow (Csikszentmihalyi, 1990), the state of peak performance where "emotions are not just contained, but positive, energized, and aligned with the task" (Goleman, 1995, p. 90). This is evidence that a person high in El will be able to face challenging situations and stay focused on the higher order priorities, in this case, safety.

Applying this line of reasoning to the application of the theory of planned behavior in a safety setting, El will influence experienced emotions, and thus impact attitudes. The quality of empathy (Freedman, 2002; Goleman, 1999) will promote considering the impact the outcome of the act will have on the other's well-being prior to performing the act. Consequential thinking (Freedman, 2002) will result in considering the potential outcomes, beneficial and adverse, short-term and long-term prior to acting. Selfregulation (Goleman, 1999) and pursuit of noble goals (Freedman, 2002) will influence the person to avoid impulsive action and to do the right thing even if it is not the politically correct or easiest thing to do. Based on the research to date, it appears that a person having greater $\mathrm{El}$ is more likely to exercise self-control and demonstrate a thought process that results in decisions supportive of a safe environment. Thus:

\section{Proposition 3: Persons with higher El will have more favorable attitudes toward safe working practices than those with lower El.}

Proposition 4: Persons with higher El will consistently perform more safely than those with lower El. 


\section{Conclusion}

This paper has reviewed progress of safety initiatives and the basic approaches taken to date. Engineering, enforcement of rules, and discipline have been drivers of significant improvements. BBS also made significant improvements. However none of the approaches to date have enabled organizations to reach the stated goal of zero accidents. The primary focus up to now has been on engineering a safe environment, training, and policy making. All of these are extrinsic factors supporting safe behavior. If we could move ahead to focusing on creating intrinsic drivers for safe behavior, further progress may be made toward reaching the stated goal of zero reportable incidents. To create an intrinsic drive to perform work in a safe manner we should examine the potential of moral maturity and El to act as intrinsic drivers supporting favorable attitudes toward safety resulting in behaviors supportive of a safe working environment.

Earlier research targeted cognitive based approaches toward improving safety and safe behavior through training, rules, and engineering a safer environment. These approaches focus primarily on constructs extrinsic to the workers. More recent approaches recognize the potential for using an affective approach calling for people based safety (Geller \& Wiegand, 2005). This represents recognition that to achieve further progress we will need to focus now on intrinsic drives for safe behavior.

By incorporating a model that specifically addresses both extrinsic (subjective norms and perceived behavioral control) and intrinsic (attitude toward the act) aspects of behavior, the theory of planned behavior (Ajzen, 1985), it is likely that more of the variability in safety-related behavior can be explained. Moral maturity and El represent constructs that are intrinsic drivers that will have an influence on safety attitudes and behavior. If it can be established empirically that these constructs have the influence that has been theorized, it is likely that both attitudes toward safety and safe behavior in the workplace can be influenced positively through training to improve El and moral maturity. This would provide industry new tools for use in advancing toward the goal of zero incidents.

The importance of this concept is that instead of taking the approach that safe behavior can be trained, required, or engineered into being, we approach it from an intrinsic point of view. Safety will improve and be sustainable if it is motivated behavior, that is, the workers do it because it is want they want to do. If it can be demonstrated that raising an individual's moral maturity and El has a positive impact on safety attitudes and behavior, it will be the first time that an approach that addresses safety-related behavior focusing primarily on intrinsic motivation has been successful. If we can create intrinsic motivation to have positive attitudes toward safety and to perform work safely, it will be sustainable over the long run and help the industry advance closer to the goal of zero reportable incidents.

The next step is to conduct field studies to ascertain whether the hypothesized relationships do indeed exist. If that is the case, a longitudinal study would be in order where a baseline for moral maturity and El would be established and then training 
would be conducted to improve moral maturity and El. Preferably the research would be conducted in an industry where safety is an issue and turnover is relatively slow such as the petrochemical industry. A study covering three to five years would be sufficient to see if the predicted effects do in fact occur.

It would be important to measure changes in moral maturity and $\mathrm{El}$ as a result of training and their respective impact on safety attitudes and behavior. In light of past research demonstrating a lack of persistence of moral maturity training (i.e. Latif, 2000, Wright, 1995;), it would be interesting to see if the same phenomenon occurs with moral maturity, El, or both in this setting. The goal is to achieve a persistent change in moral maturity and $\mathrm{El}$ to create intrinsic drivers for maintaining a safe work environment.

\section{References}

Abdolmohammadi, M., \& Sultan, J., (2002). Ethical reasoning and the use of insider information in stock trading. Journal of Business Ethics, 37(2), 165-173.

Abraham, R. (2000). The role of job control as a moderator of emotional dissonance and emotional intelligence-outcome relationships. The Journal of Psychology, 134(2), 169-184.

Ajzen, I. (1985). From intentions to actions: A theory of planned behavior. In J. Kuhl \& J. Beckmann (Eds.), Action-control: From cognition to behavior (pp. 11-39). Heidelberg: Springer.

Angelini, M. (1987). Ethical behavior for the prevention of injuries in the workplace. Ergonomics, 30, 231-237.

Bar-On, R. (2000). Emotional and social intelligence: Insights from the Emotional Quotient Inventory. In R. Bar-On \& J. D. A. Parker (Eds.), The handbook of emotional intelligence (pp. 363-388). San Francisco: Jossey-Bass.

Birkner, L., \& Birkner, R. (2000). Assessing your firm's ethical climate. Occupational Hazards, 62(10), 141-142.

Brackett, M. A., \& Mayer, J. D. (2003). Convergent, discriminant, and incremental validity of competing measures of emotional intelligence. Personality and Social Psychology Bulletin, 29(9), 1147-1158.

Ciarrochi, J., Chan, A. Y. C., \& Caputi, P. (2000). A critical evaluation of the emotional intelligence construct. Personality and Individual Differences, 28(3), 539-561.

Cellar, D. F., Nelson, C. M., \& Bauer, C. (2001). The five factor model and safety in the workplace: Investigating the relationships between personality and accident involvement. Journal of Prevention and Intervention in the Community, 22(1), 4352.

Copper, R. K., \& Sawaf, A. (1997). Executive EQ: Emotional intelligence in leadership and organization. New York: Grosset-Putnam.

Cowie, T. (2002). Rescuing the bottom line. Occupational Health \& Safety, 71(9), 90-93.

Csikszentmihalyi, M. (1990). Flow: The psychology of the optimal experience. New York: Harper \& Row.

Eckhardt, R. (2001). The moral duty to provide workplace safety. Professional Safety, $46(8), 36-39$. 
Fineman, S. (2004). Getting the measure of emotion - and the cautionary tale of emotional intelligence. Human Relations, 57(6), 719-740.

Fitch, H. G., Hermann, J., \& Hopkins, B. L. (1976). Safe and unsafe behavior and its modification. Journal of Occupational Medicine, 18(9), 624-627.

Fineman, S. (2004). Getting the measure of emotion-and the cautionary tale of emotional intelligence. Human Relations, 57(6), 719-740.

Freedman, J. (2002). What makes up El? Retrieved June 6, 2002 from www.6seconds.org.

Geller, E. S. (2001). Working safe: How to help people actively care for health and safety, $2^{\text {nd }}$ ed. New York: Lewis.

Geller, E. S., \& Wiegand, D. M. (2005). People-based safety: Exploring the role of personality in injury prevention. Professional Safety, 50(12), 28-36.

Goldstein, H. W., Zedeck, S., \& Goldstein, I. L. (2002). G: Is this your final answer? Human Performance, 15, 123-142.

Goleman, D. (1999). What makes a leader? Harvard Business Review, 76(6), 93-102.

Goleman, D. (1995). Emotional intelligence: Why it can matter more than IQ. New York: Bantam Books.

Heinrich, H. W. (1959). Industrial accident prevention: A scientific approach, $4^{\text {th }}$ ed. New York: McGraw-Hill.

Herington, C., \& Weaven, S. (2008). Improving consistency for DIT results using cluster analysis. Journal of Business Ethics, 80(3), 499-514.

Hunter, J. E., \& Hunter, R. F. (1984). Validity and utility of alternate predictors of job performance. Psychological Bulletin, 96(1), 72-98.

Huy, Q. N. (1999). Emotional capability, emotional intelligence, and radical change. Academy of Management Review, 24(2), 325-345.

Jones, T., \& Ryan, L. (1997). The link between ethical judgment and action in organizations; a moral approbation approach. Organization Science, 8(6), 663680.

Jordan, P. J., Ashkanasy, N. M., \& Härtel, C. E. J. (2002). Emotional intelligence as a moderator of emotional and behavioral reactions to job insecurity. Academy of Management Review, 27(3), 361-372.

Jordan, P. J., Ashkanasy, N. M., Härtel, C. E. J., \& Hooper, G. (2002). Workgroup emotional intelligence: Scale development and relationship to team process effectiveness and goal focus. Human Resource Management Review, 12(2), 195-215.

Kolhberg, L. (1969). Stage and sequence: The cognitive developmental approach to socialization. In D. A. Goslin (Ed.), Handbook of Socialization Theory and Research (pp. 347-480). New York: Rand McNally.

Komaki, J., Barwick, K. D., \& Scott, L. R. (1978). A behavioral approach to occupational safety: Pinpointing and reinforcing safe performance in a food manufacturing plant. Journal of Applied Psychology, 63(4), 434-445.

Krause, T. R. (2001). Second generation in behavior-based safety. Professional Safety, 46(5), 27-33.

Lam, L. T., \& Kirby, S. L. (2002). Is emotional intelligence an advantage? An exploration of the impact of emotional and general intelligence on individual performance. The Journal of Social Psychology, 142(1), 133-143. 
Latif, D. A. (2000). Ethical cognition and selection-socialization in retail pharmacy. Journal of Business Ethics, 25(4), 343-357.

Lennox, R. D., \& Wolfe, R. N. (1984). Revision of the self-monitoring scale. Journal of Personality and Social Psychology, 46(6), 1349-1364.

Martin, K. D., \& Cullen, J. B. (2006). Continuities and extensions of ethical climate theory: A meta-analytic review. Journal of Business Ethics, 69(2), 175-194.

Mayer, J. D., \& Salovey, P. (1997). What is emotional intelligence? In P. Salovey \& D. J. Sluyter (Eds.), Emotional development and emotional intelligence (pp. 3-31). New York, Basic Books.

Mayer, J. D., Caruso, D. R., \& Salovey, P. (1999). Emotional intelligence meets traditional standards for an intelligence. Intelligence, 27(4), 267-298.

Mayer, J. D., Salovey, P., \& Caruso, D. R. (1999). Models of emotional intelligence. In R. J. Sternberg (Ed.), Handbook of intelligence (pp. 396-420). Cambridge, England: Cambridge University Press.

McKendall, M., DeMarr, B., \& Jones-Rikkers, C. (2002). Ethical compliance programs and corporate illegality: Testing the assumptions of the corporate sentencing guidelines. Journal of Business Ethics, 37(4), 367-383.

Parboteeah, K., \& Kapp, E. (2008). Ethical climates and workplace safety behaviors: An empirical investigation. Journal of Business Ethics, 80(3), 515-529.

Petrides, K. V., \& Furnham, A. (2001). Trait emotional intelligence: Psychometric investigation with reference to established trait taxonomies. European Journal of Personality, 15(6), 425-448.

Reber, R. A., Wallin, J. A., \& Chhokar, J. S. (1984). Reducing industrial accidents: A behavioral experiment. Industrial Relations, 23(1), 119-125.

Rest, J. (1993). Guide to using the DIT, version 1.3. Center for the Study of Ethical Development, University of Minnesota.

Rest, J. (1994). Background: Theory, and research. In J. R. Rest and D. Navarez (Eds.), Moral Development in the Professions (pp. 1-26). Hillsdale, NJ: Erlbaum.

Salovey, P., \& Mayer, J. D. (1990). "Emotional Intelligence". Imagination, Cognition, and Personality, 9(3), 185-211.

Salovey, P. \& Slutyer, J. D. (1997). Emotional development and emotional intelligence. New York: Basic Books.

Smith, M. J., Anger, W. K., \& Ulsan, S. S. (1978). Behavioral modification applied to occupational safety. Journal of Safety Research, 10(2), 87-88.

Snyder, M. (1979). Self-monitoring processes. Advances in Experimental Social Psychology, 12, 85-128.

Sulzer-Azaroff, B., \& Austin, J. (2000). Does BBS work? Behavior-based safety and injury reduction: A survey of the evidence. Professional Safety, 45(7), 19-24.

Tidwell, A. (2000). Ethics, safety and managers. Business \& Professional Ethics Journal, 19(3-4), 161-181.

Van Rooy, D. L., \& Viswesvaran, C., (2004). Emotional intelligence: A meta-analytic investigation of predictive validity and nomological net. Journal of Vocational Behavior, 65(1), 71-95.

Van Rooy, D. L., Viswesvaran, C., \& Pluta, P. (2005). An evaluation of construct validity: What is this thing called emotional intelligence? Human Performance, 18(4), 445462. 
Weinstein, M. B. (1998). Improving behavior-based safety through TQM. Professional Safety, 43(1), 29-35.

Weisinger, H. (1997). Emotional intelligence at work: The untapped edge for success. New York: Jossey-Bass.

Weiss, H. M., \& Cropanzano, R. (1996). Affective events theory: A theoretical discussion of the structure, causes, and consequences of affective experiences at work. Research in Organizational Behavior, 18, 1-74.

Weyman, A., Clarke, D. D., \& Cox, T. (2003). Developing a factor model of coal miners' attributions on risk-taking at work. Work and Stress, 17(4), 306-320.

White Jr., R. D. (2002). Do employees act like they think? Exploring the dichotomy between moral judgment and ethical behavior. Public Administration Quarterly, 25(4), 391-412.

Wright, M. (1995). Can moral judgment and ethical behavior be learned? Management Decision, 10(17), 17-29.

Wulfson, M. (1998). Rules of the game: Do corporate codes of ethics work? Review of Business, 20(1), 12-17. 